Journal of Engineering and Applied Sciences 14 (14): 4876-4881, 2019

ISSN: 1816-949X

(C) Medwell Journals, 2019

\title{
Evaluating Web Accessibility of Spanish Universities
}

\author{
${ }^{1}$ Carlos Manez-Carvajal, ${ }^{2}$ Rocio Fernandez-Piqueras and ${ }^{2}$ Jose Francisco Cervera-Merida \\ ${ }^{1}$ Escuela de Doctorado, \\ ${ }^{2}$ Universidad Catolica de Valencia San Vicente Martir, C/Sagrado Corazon 5, 46110 Godella, Spain
}

\begin{abstract}
The objective of this study is to evaluate web accessibility of the home pages of educational platforms of the top 20 Spanish universities found on the university ranking webometrics. The analysis has been carried out on the basis of the international standard set by the World Wide Web consortium. The above is a legal requirement in Spain for the public administration and for websites financed partially or fully by the government as well as those that belong to big companies. Various automatic web accessibility evaluation tools have also been used apart from manual verifications. The study reveals that the most prestigious Spanish universities hardly comply with the regulations of web accessibility.
\end{abstract}

Key words: Web accessibility, university, disability, WCAG 2.0 , W3C, World Wide Web consortium

\section{INTRODUCTION}

We live in the age of Information and Communications Technology (ICT) and in the digital university model (Orueta and Pavon, 2008) an online educational model wherein universities offer new opportunities to people who experience difficulties accessing higher education.

This educational model is very much in line with the mission statement of the United Nations (UNESCO) in terms of education which is to provide quality education and lifelong learning for all.

Practically all universities offer virtual spaces to develop teaching as well as learning with the support of the constant development of new technologies. For Adell (1997) "Online learning is an emerging way used to provide knowledge and skills to a wide variety of segments of the population, so that, people with difficulties of access to face to face educational institutions could also benefit from education". Ortega hinted at these learning scenarios in different ways when speaking about distance learning, cyber-schools or online universities.

The online university has been evolving as fast as new technologies. It turns out to be extraordinarily favored by the expansion of the internet and the proliferation of cutting-edge technological devices. As a result, university studies which fail to include a virtual component are perceived as strange and outside of the norm. Moreover, the contemporary university is not only concerned with young students but also adapts itself to the educational needs of adults and professionals of the business world (Fernandez, 2010). We can be said to live mainly in an internet society in which space and distance are no longer a limitation.

Nonetheless, one must take into account that not all users access the web with the same technical skills. The elderly and people with disabilities sometimes use assistive technology support in order to access and interact with online content. Designing and codifying web content in the right way facilitates the interaction with assistive technology and allows people with disabilities to use the web without limitations, taking advantage of the opportunities it provides.

The entity which looks after the proper development of the web is the World Wide Web Consortium (W3C) which defines web accessibility as "A universal access to the web, designed to work for all people whatever their hardware, software, language, location or ability" (Anonymous, 2018a-c).

With the aim of preserving the rights of all, the W3C created the Web Accessibility Initiative (WAI), a work team in charge of establishing the norms of accessibility to online contents, called Web Content Accessibility Guidelines (WCAG). Their goal is to guide web design towards one which is more universal, reducing the existing barriers and trying to make the contents accessible for the highest number of people possible.

This international standard for web accessibility is a legal requirement in Spain and public administration is subject to it (De Normalizacion, 2012) and what is more, the convention on the rights of persons with disabilities states in Article 24.5 that "Parties shall ensure that persons with disabilities are able to access general tertiary

Corresponding Author: Carlos Manez Carvajal, C/Merida el Humano 25, 1246008 Valencia, Spain 
education, vocational training, adult education and lifelong learning without discrimination and on an equal basis with others" (UN., 2014).

Principles and directives of the WCAG 2.0: WCAG continues to evolve, the most recent Version being 2.0 which gathers the main norms and principles, verifiable conformity criteria as well as recommended techniques (Anonymous, 2018a). Those principles are as follows:

- Perceivable: "information and user interface components must be presentable to users in ways they can perceive"

- Operable: "user interface components and navigation must be operable"

- Understandable: "information and the operation of user interface must be understandable"

- Robust: "content must be robust enough that it can be interpreted reliably by a wide variety of user agents, including assistive technologies"

Below these principles we can find guidelines which are divided into verification criteria being, thus, classified into three conformance levels: A, AA and AAA (priority 1-3) according to, their level of importance and requirements in terms of the accessibility of contents.

Priority 1 and 2 errors seriously affect the use of the web by users with disabilities and they should be reviewed and corrected in order to meet the current regulation.

Barriers and difficulties of access: According to, Brajnik (2006) a web barrier is any condition that makes it difficult for people to reach an objective while visiting a website through assistive technology.

The blind or those with serious visual impairment interact with the web content through screen readers, computer programs capable of transforming the textual content into voice. Thus, the creators of online content should provide a description of images and multimedia elements, making them easier to access. In addition an adequate contrast is necessary for all users, especially, users with low vision or individuals who cannot differentiate between certain colors (WebAIM, 2018).

People with motor impairment may interact with the online content using specific hardware and software such as especially, designed keyboards and mice. They may need more time to type or interact with web content for this reason it is fundamental to provide them with full keyboard support, adding large areas on which to click, offering them enough time to complete a task and designing a simple web structure (Anonymous, 2018b).
A website should contain alternative textual content like subtitles and transcriptions of videos, sounds and multimedia presentations, all of which are fundamental aspects for a person with a hearing impairment.

People with cognitive, learning and neurological disabilities may have difficulty using a website with complex navigation mechanisms or page layouts which are difficult to understand and use. Therefore, a simple design of each website using images, clear textual content and simple navigation mechanisms would be highly recommended (Anonymous, 2018b).

Web designers and content creators should take into account both the elderly and people with functional diversity into account when designing a website. Taking the users into consideration during the website developing process implies understanding them, discovering their needs and asking about their user experience in order to improve it (Montero and Fernandez, 2013).

\section{MATERIALS AND METHODS}

In order to carry out this kind of study, it is possible to use software tools which help to evaluate the accessibility level of a website. These tools detect barriers and difficulties that users might have when accessing a website but they cannot fully determine whether or not a website is accessible. To that end, human evaluation is required (Mascaraque et al., 2009).

There have been numerous studies on the subject of web accessibility of educational university platforms, portals or e-Learning platforms, both at national and international levels (Caballero-Cortes et al., 2009; Chacon-Medina et al., 2013; Hilera et al., 2013; Ismailova and Inal, 2018; Ismailova and Kimsanova 2017; Nir and Rimmerman, 2018).

In all of these previous studies, various methodologies and automatic tools were used to carry out the evaluation of web accessibility of university websites. The principles, guidelines and success criteria as established by the WCAG 2.0 were tested by Chacon-Medina et al. (2013) manually and using automated evaluation tools and revealed that not even one of the 74 Spanish universities fully met the web accessibility requirements. In the same year Hilera et al. (2013) carried out a comparative study, analyzing Spanish universities and those from other countries, the former ones showing lower accessibility levels than the latter. There exist similarities between both studies such as the selection of home pages of university platforms as well as the selection of automated evaluation tools to carry out their research. 
Sample: As the study shows, we have chosen 20 top Spanish universities from the ranking Webometrics as our sample. Webometrics measures the online presence and visibility of the said institutions. We decided to subject the home pages of the websites to analysis, just like the studies (Espadinha et al., 2011; Ismail and Kuppusamy, 2018; Laitano, 2015) had done, since, we consider the home page to be the most representative and because webmasters pay it the most attention.

Tools: To begin the analisys, we also used source code validators recommended by the $\mathrm{W} 3 \mathrm{C}$ with the aim of checking the HTML code and CSS style sheets. In the second step of the process, WAVE is used to evaluate color combinations in the foreground and the background color of the visual presentation.

Finally, we used Web Accessibility Test (TAW) in the same way in which it had been used by previous research focused on the evaluation of web accessibility of Spanish universities (Chacon-Medina et al., 2013; Hilera et al., 2013). TAW is an application designed by Center for the development of Information and Communication Technologies (CTIC). It runs an analysis of the website according to, the web content accessibility guidelines and provides a detailed report of errors and warnings found on every page. The organization of this information is based on four fundamental principles of web accessibility (Perceivable, Operable, Understandable, Robust).

\section{RESULTS AND DISCUSSION}

W3C validators: We used validators recommended by the W3C and checked the syntax HTML and CSS of each university home page as can be observed in Table 1 . The data shows that all the home pages of the selected universities contain HTML errors and that some of these errors are quite serious such as those shown by University of Valencia $(n=848)$. Error free source code would facilitate the interaction of web content with assistive technology.

WAVE: We analyze the website using the WAVE tool in order to check foreground color and the color of the visual presentation. The results of the evaluation of all the university websites can be observed in Table 2 .

The WAVE tool shows that only in Complutense University of Madrid is the contrast ratio correct. The results as shown by WAVE reveal that University of Valencia $(\mathrm{n}=74)$, University of Zaragoza $(\mathrm{n}=70)$ and Pompeu Fabra University $(n=68)$ display the highest number of contrast errors, coinciding with the analysis
Table1: Source code errors found by W3C validators

\begin{tabular}{lcc}
\hline Institutions & $\begin{array}{c}\text { W3C markup } \\
\text { validation service }\end{array}$ & $\begin{array}{c}\text { W3C CSS } \\
\text { validation service }\end{array}$ \\
\hline University of Barcelona & 17 & 13 \\
Complutense University of Madrid & 4 & 12 \\
University of Granada & 10 & 4 \\
University of Valencia & 848 & 10 \\
Autonomous University of Barcelona & 35 & 13 \\
Autonomous University of Madrid & 96 & 0 \\
Polytechnic University of Catalonia & 19 & 6 \\
Polytechnic University of Valencia & 35 & 12 \\
The Technical University of Madrid & 25 & 1 \\
Pompeu Fabra University & 38 & 410 \\
University of Sevilla & 6 & 0 \\
University of Zaragoza & 30 & 11 \\
University of the Basque Country & 14 & 389 \\
University of Santiago de Compostela & 10 & 2 \\
University of Murcia & 8 & 10 \\
University of Malaga & 5 & 11 \\
University of Oviedo & 2 & 13 \\
University of Salamanca & 148 & 6 \\
Carlos III University of Madrid & 13 & 33 \\
University of Castilla-La Mancha & 46 & 2
\end{tabular}

Table 2: Contrast errors found on home pages by WAVE

Institutions

University of Barcelona

Complutense University of Madrid

University of Granada

University of Valencia

Autonomous University of Barcelona

Autonomous University of Madrid

Polytechnic University of Catalonia

Polytechnic University of Valencia

The Technical University of Madrid

Pompeu Fabra University

University of Sevilla

University of Zaragoza

University of the Basque Country

University of Santiago de Compostela

University of Murcia

University of Malaga

University of Oviedo

University of Salamanca

Carlos III University of Madrid

University of Castilla-La Mancha

Contrast errors

8

8
0

31
74

22

40

39

4
8
68

68

12

70

11
26

14

12

9

9

1

provided by TAW. These contrast errors may be a barrier of access for users with low vision or individuals who cannot differentiate between certain colors.

TAW: The results provided by TAW show the existence of barriers of access to information for the elderly and those with disability in the chosen websites as shown in Table 3.

The automatic evaluation tool, TAW shows that all these online learning platforms display web accessibility errors on their home pages. The lowest results in terms of meeting the regulation have been obtained by University of Valencia $(\mathrm{n}=225)$ Pompeu Fabra University $(\mathrm{n}=194)$ and University of Zaragoza $(n=118)$. When analyzing the results according to, principles, we found that the failure to meet requirements mainly resides in the perceivable principle (66\%) as can be observed in Fig. 1. 
Table 3: Web accessibility errors on home pages of university websites, categorized by principle

\begin{tabular}{|c|c|c|c|c|c|}
\hline Institutions & Perceivable & Operable & Understandable & Robust & Total \\
\hline University of Barcelona & 10 & 8 & 0 & 0 & 18 \\
\hline Complutense University of Madrid & 2 & 7 & 0 & 1 & 10 \\
\hline University of Granada & 35 & 0 & 0 & 3 & 38 \\
\hline University of Valencia & 156 & 10 & 11 & 48 & 225 \\
\hline Autonomous University of Barcelona & 25 & 15 & 0 & 11 & 51 \\
\hline Autonomous University of Madrid & 56 & 0 & 1 & 8 & 65 \\
\hline Polytechnic University of Catalonia & 39 & 2 & 0 & 2 & 43 \\
\hline Polytechnic University of Valencia & 20 & 1 & 0 & 0 & 21 \\
\hline The Technical University of Madrid & 13 & 7 & 0 & 0 & 20 \\
\hline Pompeu Fabra University & 140 & 44 & 3 & 7 & 194 \\
\hline University of Seville & 25 & 0 & 0 & 4 & 29 \\
\hline University of Zaragoza & 91 & 22 & 1 & 4 & 118 \\
\hline University of the Basque Country & 19 & 0 & 0 & 9 & 28 \\
\hline University of Santiago de Compostela & 32 & 0 & 0 & 31 & 63 \\
\hline University of Murcia & 17 & 0 & 0 & 20 & 37 \\
\hline University of Málaga & 27 & 22 & 0 & 3 & 52 \\
\hline University of Oviedo & 9 & 3 & 0 & 4 & 16 \\
\hline University of Salamanca & 13 & 1 & 0 & 36 & 50 \\
\hline Carlos III University of Madrid & 6 & 0 & 1 & 5 & 12 \\
\hline University of Castilla La Mancha & 1 & 18 & 2 & 3 & 24 \\
\hline
\end{tabular}

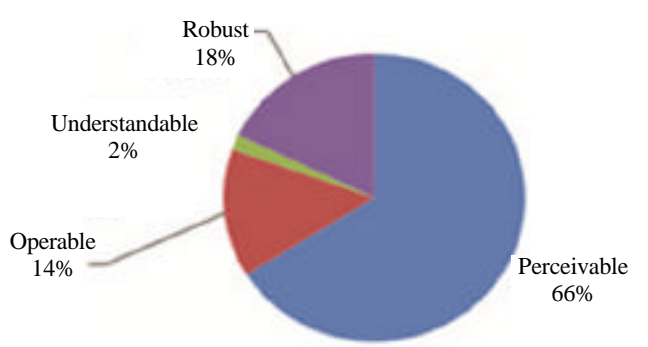

Fig. 1: Total error categorized by principal (web accessibility error)

With regard to the perceivable principle, TAW detects the omission of textual alternatives to non-textual contents ( $\mathrm{f}=104$ ) or the cases when their description is inadequate. The textual alternatives are fundamental for a person who interacts with the web using a screen reader. Pertaining to the operable principle, the error most often found is missing text in the links ( $\mathrm{f}=279$ ) which makes it easier for users to know where the links are and where they lead. As to the understandable principle, the most common error was encountered in form controls. Finally, results regarding the Robust principle are mostly due to source code errors which are detrimental to the interaction between the website content and assistive technology.

After classifying web accessibility errors by priority Fig. 2, we discovered that around $60 \%$ are errors of priority 1 which is a basic requirement that developers must meet. The $40 \%$ of the errors belong to priority 2 and should be rectified in order to facilitate access to a certain group of users. The detected errors, categorized by priority as well as university institution are shown in Fig. 3.

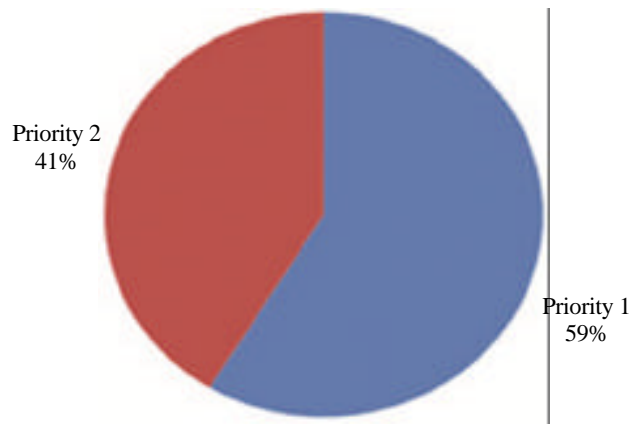

Fig. 2: Errors categorized by priority

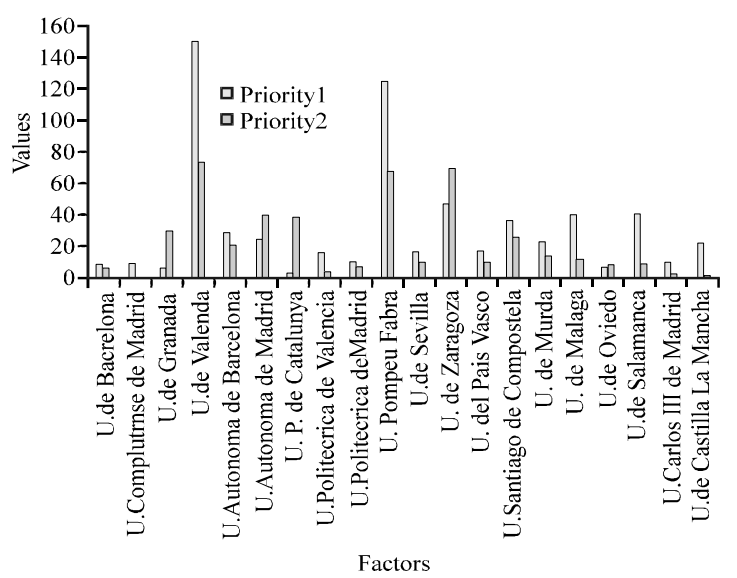

Fig. 3: Web accesibility errors catergorized by piority, the order of web matericsranking is maintained

Errors and possible solutions: After analyzing the data by principle, it is the perceivable principle which displays the highest number of web accessibility errors. The websites should include an alternative description using alt attribute whenever it is necessary. Using tools such as 
Wave to check foreground color and the color of the visual presentation would reduce the existing barriers for users with low vision. In reference to the operable principle, adding alternative texts to links would solve a great number of these errors. In relation to the understandable principle, the adequate use of the label element in the forms is necessary. Finally, with regard to the Robust principle, many errors are caused by the inadequate use of HTML grammar when designing a website. The use of validators recommended by the W3C would eliminate most of these errors and would create a better interaction with assistive technology, thus, improving the user experience.

The analysis provided by the automated tools is of great significance and reveals that the none of the selected websites have met the regulation fully.

The main objective of this research has been to analyze the degree to which the top 20 Spanish universities, according to, the ranking Webometrics, meet the WCAG 2.0 regulation through the use of automated tools.

The results obtained shows that none of the selected websites have met the regulation, just as in the study carried out by Chacon-Medina et al. (2013). The study concludes that the main problems shown by websites lie in how the information is perceived by users as well as difficulties in the use of the website. These difficulties are heightened in the case when the user is a person with disabilities using assistive technology. As the W3C indicates, the use of this kind of automated tools is recommended in order to detect and troubleshoot the failure to meet the regulation as proven by the analysis. These tools must not substitute user tests. They are simply an excellent complementary tool at an expert's disposal.

\section{CONCLUSION}

The WCAG 2.0 guidelines are meant to protect the rights of people when it comes to information access and this study proves the existence of barriers experienced by the elderly and people with disabilities when using university websites. The online university is an opportunity to improve the education of all people and to reduce inequality among individuals. Undoubtedly, the best method to attain this is by facilitating universal access to information, thus, fostering and achieving a truly inclusive education.

\section{REFERENCES}

Adell, J., 1997. [Trends in education in the society of information technologies (In Spanish)]. Edutec. Electron. Mag. Educ. Technol., 7: 1-21.
Anonymousa, 2018a. Accessibility principles. World Wide Web Consortium, USA. https://www.w3.org/ WAI/ fundamentals/accessibility-principles/

Anonymousb, 2018b. Diverse abilities and barriers. Web Accessibility Initiative, USA. https://www.w3.org/ WAI/people-use-web/abilities-barriers/

Anonymousc, 2018c. Introduction to web accessibility. World Wide Web Consortium, USA. https://www. w3.org/WAI/fundamentals/accessibility-intro/

Caballero-Cortes, L., C. Faba-Perez and F.D. Moya-Anegon, 2009. [Comparative evaluation of the accessibility of the web spaces of the Spanish and North American university libraries (In Spanish)]. Lib. Res., 23: 45-66.

Chacon-Medina, A., H. Chacon-Lopez, M.D. Lopez-Justicia and C. Fernandez-Jimenez, 2013. [Difficulties in Web Accessibility of Spanish Universities according to the WCAG 2.0 Standard (In Spanish)]. Spanish Mag. Sci. Doc., Vol. 36,

De Normalizacion, A.E., 2012. [UNE 139803: Accessibility requirements for web content]. Spanish Association for Standardization, Madrid, Spain. http://travesia.mcu.es/portalnb/jspui/handle/10421/ 6861

Espadinha, C., L.M. Pereira, F.M. Da Silva and J.B. Lopes, 2011. Accessibility of Portuguese Public Universities sites. Disability Rehabil., 33: 475-485.

Fernandez, R., 2010. [Background factors in the use of virtual training environments and their effect on teacher performance]. Ph.D Thesis, Universitat Politecnica de Valencia, Valencia, Spain. (In Spanish)

Hilera, J.R., L. Fernandez, E. Suarez and E.T. Vilar, 2013. [Evaluation of the accessibility of web pages of Spanish and Foreign universities included in international university rankings (In Spanish)]. Spanish Mag. Sci. Doc., 36: 1-16.

Ismail, A. and K.S. Kuppusamy, 2018. Accessibility of Indian universities homepages: An exploratory study. J. King Saud Univ. Comput. Inf. Sci., 30: 268-278.

Ismailova, R. and G. Kimsanova, 2017. Universities of the Kyrgyz Republic on the web: Accessibility and usability. Universal Access Inf. Soc., 16: 1017-1025.

Ismailova, R. and Y. Inal, 2018. Accessibility evaluation of top university websites: A comparative study of Kyrgyzstan, Azerbaijan, Kazakhstan and Turkey. Universal Access Inf. Soc., 17: 437-445.

Laitano, M.I., 2015. [Web accessibility in the Argentine University public space (In Spanish)]. Spanish Mag. Sci. Doc., 38: 1-9.

Mascaraque, E.S., A.M. Ocana and I.O. Martos, 2009. [Technical guidelines related to web accessibility (In Spanish)]. An. Doc., 12: 255-280. 
Montero, Y.H. and F.J.M. Fernandez, 2013. [Proposal to adapt the user-centered design methodology for the development of accessible websites (In Spanish)]. Spanish J. Sci. Doc., 27: 330-344.

Nir, H.L. and A. Rimmerman, 2018. Evaluation of web content accessibility in an Israeli institution of higher education. Universal Access Inf. Soc., 17: 663-673.
Orueta, J.L. and L.M. Pavon, 2008. [White Paper of the Digital University 2010]. Vol. 11, Telefonica Foundation, Madrid, Spain, Pages: 313 (In Spanish). UN., 2014. [Convention on the Rights of People with Dsabilities]. In: The Major International Treaties on Human Rights, UN. (Ed.). United Nations, New York, USA., pp: 271-310.

WebAIM., 2018. We have web accessibility in mind. WebAIM, Logan, Utah, USA. https://webaim.org/ 